\title{
Outcome Evaluation of Gabapentin as Add-on Therapy for Partial Seizures
}

\author{
Joseph Bruni on behalf of the "NEON" Study Investigators Group $\dagger$
}

\begin{abstract}
Objective: The safety, tolerability, efficacy, and impact on quality of life of gabapentin (Neurontin $\left.{ }^{\circledR}\right)$ as adjunctive therapy to carbamazepine (CBZ) and/or phenytoin (PHT) was assessed in epileptic patients with partial seizures. Methods: NEON (Neurontin Evaluation of Outcomes in Neurological Practice) was an open-label, prospective, multicentre study conducted in patients on a stable dose of $\mathrm{CBZ}$ and/or PHT and experiencing an average of up to 4 complex partial seizures with or without secondary generalization per month, with no seizure-free months. The treatment lasted 20 weeks. Gabapentin was started at $400 \mathrm{mg} /$ day and was individually titrated to effective tolerable dose up to $2400 \mathrm{mg} /$ day. Quality of life was evaluated using the QOLIE-10 questionnaire. Results: A total of 141 patients were enrolled at 36 sites; 114 patients were evaluable for efficacy analyses. The mean maintenance dose of gabapentin was 1600 mg/day (range $=300-3200$ ). A decrease of $50 \%$ or more in frequency of complex partial + secondarily generalized seizures was observed in 81 $(71 \%)$ patients $(\mathrm{p}=0.0001)$. Fifty two $(46 \%)$ patients were seizure-free during the last 8 weeks of treatment. A significant improvement ( $p$ $<0.05)$ was observed in 5 of the 10 questions of the QOLIE-10, as well as in the composite QOL score $(p=0.0002)$. The most frequent adverse events included somnolence (16\%), dizziness $(9 \%)$, and asthenia $(6 \%)$. Twenty-five $(18 \%)$ patients prematurely discontinued the study, $16(11 \%)$ of them due to adverse events. Conclusions: This study indicates that treatment with gabapentin as adjunctive therapy to standard antiepileptic drugs in this group of patients not only provides significant improvement in seizure control, but also has a positive impact on quality of life. The clinical benefits in efficacy, safety and tolerability demonstrated at 20 weeks are sustained, and no tolerance develops with gabapentin in longer term use.
\end{abstract}

RÉSUMÉ: Évaluation de l'efficacité de la gabapentine comme traitement adjuvant des crises d'épilepsie partielles. Objectif: Nous avons évalué la sécurité, la tolérabilité, l'efficacité et l'impact sur la qualité de vie de la gabapentine (Neurontin®) comme traitement adjuvant à la carbamazépine (CBZ) et/ou à la phénytöne (PHT) chez les épileptiques qui ont des crises partielles. Méthodes: L'étude NEON (Neurontin Evaluation of Outcomes in Neurological Practice) était une étude multicentre, prospective, ouverte, chez des patients prenant une dose stable de CBZ et/ou de PHT et qui présentaient en moyenne jusqu'à 4 crises partielles complexes avec ou sans généralisation secondaire par mois, sans période de un mois exempte de crise. La durée du traitement était de 20 semaines. La gabapentine était prescrite d'abord à $400 \mathrm{mg} /$ jour et la posologie était individualisée jusqu'à la dose tolérée ou maximum de $2400 \mathrm{mg} / \mathrm{jour}$. La qualité de vie était évaluée au moyen du questionnaire QOLIE-10. Résultats: Au total 141 patients ont été inclus dans l'étude dans 36 centres; les analyses d'efficacité ont pu être faites sur 114 patients. La dose moyenne d'entretien de gabapentine était de $1600 \mathrm{mg} / \mathrm{jour}$ (intervalle de 300 à 3200). Une diminution de $50 \%$ ou plus dans la fréquence des crises complexes partielles + et secondairement généralisées a été observée chez 81 $(71 \%)$ des patients $(p=0.0001)$. Cinquante-deux patients $(46 \%)$ n'ont eu aucune crise pendant les 8 dernières semaines de traitement. Une amélioration significative $(p<0.05)$ a été observée dans la réponse à 5 des 10 questions du QOLIE-10, ainsi que dans le score composé de QOL $(p=0.0002)$. Les effets secondaires les plus fréquents étaient la somnolence (16\%), les étourdissements (9\%) et l'asthénie (6\%). Vingt-cinq (18\%) des patients ont quitté l'étude prématurément, $16(11 \%)$ à cause d'effets secondaires. Conclusions: Cette étude indique que la gabapentine comme adjuvant des médications antiépileptiques standards procure non seulement une amélioration significative du contrôle des crises mais a aussi un effet positif sur la qualité de vie. Les bénéfices cliniques d'efficacité, de sécurité et de tolérabilité démontrés à 20 semaines sont soutenus et les patients ne développent pas de tolérance à la gabapentine à plus long terme.

Can. J. Neurol. Sci. 1998; 25: 134-140

Over the past few years, numerous new anticonvulsant drugs have become available for the treatment of various seizure types. ${ }^{1-4}$ Among these, gabapentin (Neurontin $($ ) ) has been introduced recently in Europe and North America as adjunctive therapy for the management of patients with partial epilepsy who are not satisfactorily controlled by conventional therapy. Although the precise mechanism of action of gabapentin remains to be defined, it appears to be distinctly different from that of other AEDs. ${ }^{5,6}$

Controlled studies have demonstrated that gabapentin reduces the frequency by $50 \%$ or more, of complex partial seizures (CPS) and secondarily generalized seizures (SGS), in $26 \%$ and $54 \%$ of epileptic patients, respectively. ${ }^{3,7,8}$
In earlier clinical efficacy trials, gabapentin has been evaluated predominantly in combination with other AEDs, as a third or fourth add-on drug, in patients refractory to available AEDs and with a long history of epilepsy. These represent some of the most difficult-to-treat patients. The aim of this study was to characterize the therapeutic potential of gabapentin as adjunctive therapy in patients seen in outpatient, neurological practice. The safety, tolerability and efficacy of gabapentin was assessed

From the NEON Study Investigators Groupt (see Appendix).

RECEIVED MAY 14, 1997. ACCEPTED IN FINAL FORM NOVEMBER 28, 1997

Reprint requests to: Joseph Bruni, The Wellesley Hospital, E. K. Jones Building, 318 160 Wellesley Street East, Toronto, Ontario, Canada M4Y $1 \mathrm{~J} 3$ 
in epileptic patients with CPS with or without SGS, who were not adequately controlled and considered suitable for additional medication to existing CBZ and/or PHT therapy. Some patients also experienced simple partial seizures (SPS). The introduction of an AED to the patient's current treatment for epilepsy must take into account not only its efficacy and safety profile but also the overall performance of patients in their daily activities. Therefore, this study also evaluated quality of life parameters for patients given Neurontin $®$ as first line adjunctive therapy to standard AEDs.

\section{Methods AND Materials}

\section{Study Design}

This was an open-label, prospective, multicentre trial conducted at 36 centres located across Canada. The study consisted of a 20-week treatment period, with evaluations at baseline visit and following 4, 8, 12 and 20 weeks of treatment. Every effort was made to conduct a final evaluation in patients who discontinued treatment prematurely.

In order to evaluate the use of gabapentin in general neurological practice, minimal interference was exerted with regards to normal office/clinic procedures for the management of epilepsy. The collection of information on seizure frequency was elicited in the same manner at both the baseline visit and the final visit (endpoint evaluation), without the use of diaries or aids other than those considered standard for each individual patient, investigator or Institution.

\section{Patient Population}

One hundred and forty-one adult patients (53 male, 88 female) aged $18-88$ years were enrolled in the study. Patients were diagnosed with partial epilepsy, according to the Commission on Classification and Terminology of the International League against Epilepsy. ${ }^{9}$ All patients had a history of up to 8 CPS with or without SGS during the two months preceding the baseline visit, with no seizure-free months during that interval; SPS were experienced by some patients. They had to be on a stable dose of either CBZ, PHT or both for at least 30 days prior to study entry, with no AED(s) other than CBZ or PHT within two months preceding study entry. Currently available formulations of CBZ were permitted. Female patients who were pregnant or nursing were excluded, while female patients considered sexually active had to be practicing a reliable method of contraception in order to participate in this trial.

Patients with primary generalized seizures, pseudo-seizures, or non-epileptic seizures were excluded. The occurrence of status epilepticus within six months prior to study entry, a history of progressive structural CNS lesion or progressive encephalopathy, or the use of any investigational medication within the two months preceding study entry also precluded patients from enrollment. A signed informed consent was obtained from each patient prior to the initiation of the study. The protocol and informed consent form were approved by an independent Central Institutional Review Board, and by local IRB/Ethics Committees where appropriate.

\section{Study Medication and Dosage Adjustments}

Gabapentin was supplied as the market formulation of Neurontin ${ }^{\circledR}$ (400 $\mathrm{mg}$ capsules) and used according to the guidelines of the current Product Monograph. The treatment was initiated at $400 \mathrm{mg} /$ day, followed by a 2 -week titration at increments of $400 \mathrm{mg} / \mathrm{day}$, up to a base maintenance dose of $1200 \mathrm{mg} / \mathrm{day}$, unless not tolerated by the patient. Further titration was achieved on an individual basis, based on the response and the judgment of the clinician, up to the recommended dose of $2400 \mathrm{mg} / \mathrm{day}$. Response was evaluated at each interim visit based on seizure frequency and tolerability. Attempts were made to titrate gabapentin up to an effective tolerable dose before considering the addition of other AEDs. Assessment of compliance with the study medication prescribed was based on patient interviews during the study visits.

Concomitant AEDs, including CBZ and PHT, were used under the guidelines outlined in their respective Product Monographs.

\section{Study Assessments}

The efficacy assessments were based on the frequency of CPS, SGS and SPS. Frequency of these seizures was assessed during the eight weeks preceding the baseline visit and during the last eight weeks of treatment with gabapentin, as well as at each interim visit. Seizure frequencies were calculated as the number of seizures per four-week interval. The efficacy of gabapentin was evaluated using the responder rate, which represented a reduction of $50 \%$ or more in seizure frequency from baseline to endpoint. The responder rate was determined for the combined frequency of CPS and SGS (primary efficacy parameters) and for the individual frequencies of CPS, SGS and SPS (secondary efficacy parameter).

The safety assessments included the recording of adverse events throughout the study and a brief neurological examination and clinical laboratory tests at the beginning and the end of the treatment. An overall assessment of tolerability to gabapentin was conducted by the investigators at the end of the study.

Quality of life assessments were conducted using the QOLIE-10 questionnaire ${ }^{10}$ administered to patients at both baseline and the endpoint evaluation. Scores ranging from 1 to 5 were recorded for each of the 10 individual questions. In addition, a composite score, based on the combination of the scores obtained for the 10 individual questions, was calculated to obtain the "Composite QOL Score".

\section{Data Analyses}

The analyses of all demographic and safety data were based on the intent-to-treat patient population, that is, all patients who received at least one dose of gabapentin. The analysis of the efficacy parameters was based on the population of evaluable patients, which included all patients who completed at least eight weeks of treatment and for whom no major protocol violations were observed. These major protocol violations included the use of any AED other than C.BZ and/or PHT at study entry, or the occurrence of more than eight CPS + SGS during the eight weeks preceding the baseline visit.

All inferential statistical analyses were based on two-tailed tests, with a value of 0.05 defined as the minimum level of significance. Unless otherwise stated, data are presented as means \pm S.D. 


\section{ReSUltS}

\section{Patient Population}

A total of 141 patients were enrolled in the study. Information on the patients' demographics and history of epilepsy is presented in Table 1 . The majority of patients $(67 \%)$ were 45 years of age or less, while $8 \%(\mathrm{~N}=11)$ were over 65 years old. Most of the patients (74\%) had epilepsy for more than 10 years. As shown in Table 1, the etiology of epilepsy was unknown for the majority of the patients. Approximately $79 \%(\mathrm{~N}=111)$ of the patients had previously received at least one AED other than their current treatment at study entry (Table 2 ).

At study entry, $88(62 \%)$ patients were being treated with CBZ as single therapy at a mean dose of $990 \pm 320 \mathrm{mg} /$ day (range 400-2000), while 31 (22\%) were on PHT monotherapy at

Table 1: Summary of Patients and Causes of Epilepsy for all Patients Entered into the Study $(\mathrm{N}=141)$.

\begin{tabular}{lrl}
\hline & Number ( S.D. (range) \\
\hline Age & $42 \pm 14$ & years (18-80) \\
Age at First Diagnosis of Epilepsy & $21 \pm 16$ & years $(1-77)$ \\
Duration of Epilepsy at Study Entry & $20 \pm 13$ & years $(1-70)$ \\
\hline & Number of patients (\%) \\
\hline Gender: - Male & $53(38 \%)$ \\
- Female & $88(62 \%)$ \\
Cause of Epilepsy* \# & & \\
- Unknown & $76(54 \%)$ \\
- Congenital & $14(10 \%)$ \\
- Head trauma & 13 & $(9 \%)$ \\
- Infection & 13 & $(9 \%)$ \\
- Febrile & 8 & $(6 \%)$ \\
- Vascular & 8 & $(6 \%)$ \\
- Birth complications & 7 & $(5 \%)$ \\
- Other & 6 & $(4 \%)$ \\
- Family history & 4 & $(3 \%)$ \\
\hline
\end{tabular}

*More than one cause of epilepsy may be assigned to some patients. \#Reference \#9.

Table 2: Summary of Prior Treatment with Antiepileptic Drugs (AED).

\begin{tabular}{lc}
\hline Previous Treatment & Number of Patients (\%) \\
\hline No Previous AED & $\mathbf{3 0}(\mathbf{2 1 \% )}$ \\
\hline Previous AED* & $\mathbf{1 1 1 ( 7 9 \% )}$ \\
\hline Phenytoin & $49(35 \%)$ \\
Phenobarbital & $38(27 \%)$ \\
Clobazam & $31(22 \%)$ \\
Valproic Acid & $28(20 \%)$ \\
Primidone & $27(19 \%)$ \\
Carbamazepine & $17(12 \%)$ \\
Other $\dagger$ & $23(16 \%)$ \\
\hline
\end{tabular}

*Patients may have received more than one AED. †Includes clonazepam (3), gabapentin (1), phelantin (1), vigabatrin (7), methsuximide (3), ethosuximide (3), tiagabine (1), diazepam (1), and five cases not specified. an average dose of $330 \pm 80 \mathrm{mg} /$ day (range 100-500). Twentytwo $(16 \%)$ patients were receiving both CBZ and PHT at a mean daily dose of $1040 \pm 510$ (range 400-2400) and $330 \pm 110 \mathrm{mg}$ (range 100-600), respectively.

The patient disposition flow chart between study entry and the completion of the trial, is shown in Figure 1. Out of the 141 patients enrolled (intent-to-treat population), 25 (18\%) discontinued treatment prior to the completion of the 20 -week treatment interval. Of these, $16(11 \%)$ discontinuations were attributed to adverse events, $3(2 \%)$ to inadequate seizure control, $1(1 \%)$ to non-compliance, $3(2 \%)$ to loss to follow-up, and $2(1 \%)$ to other, unspecified reasons. Out of the 25 discontinued patients, seven had completed eight weeks or more of treatment and were considered as evaluable patients, whereas nine of the patients who completed the 20-week gabapentin treatment period were excluded from the efficacy analysis due to major protocol violations, leading to a total of 114 evaluable cases.

\section{Efficacy Evaluations}

More than $25 \%$ of the patients reported SGS or SPS in addition to CPS at study entry. The respective frequencies of individual seizure types at baseline by the 114 evaluable patients are presented in Table 3.

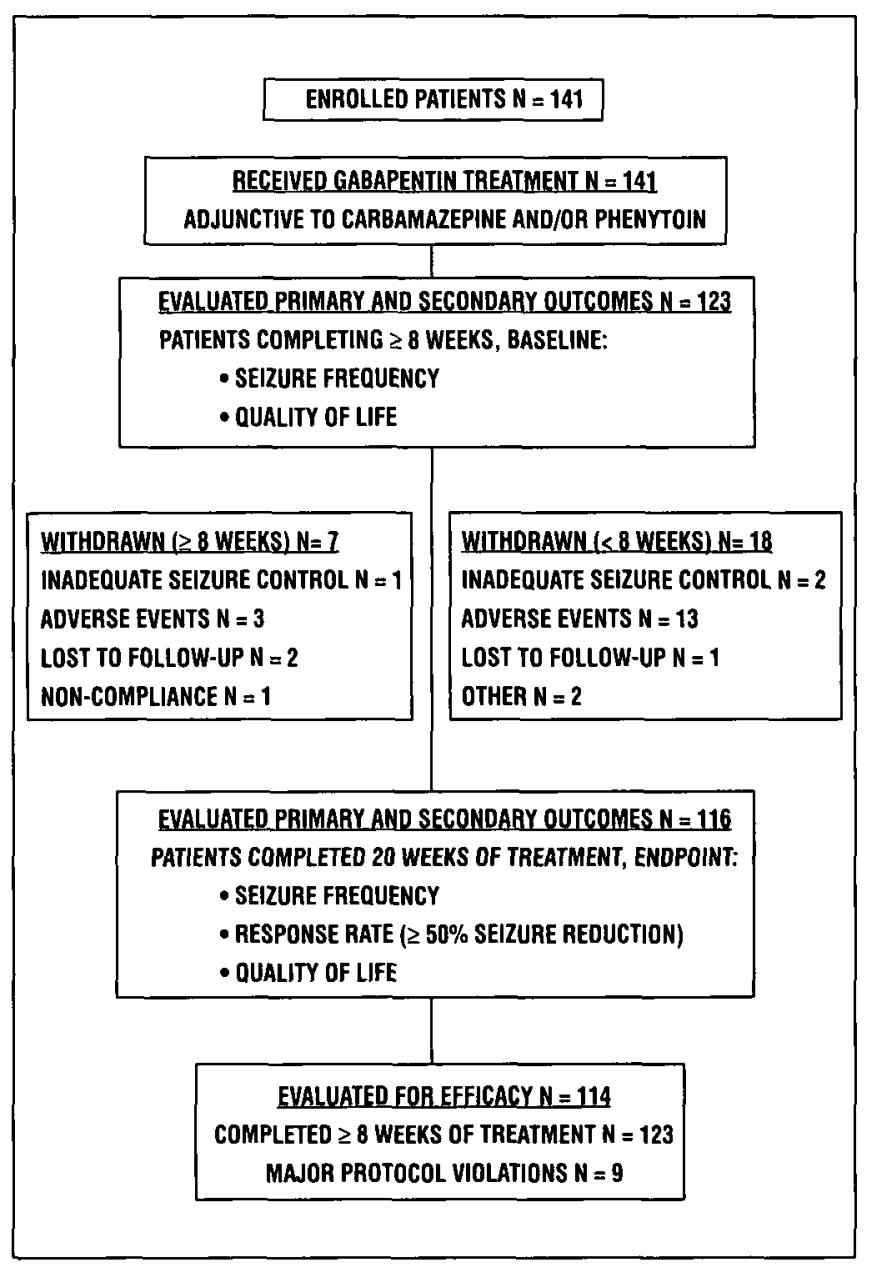

Figure 1: Summary of Patients Enrolled and Followed-up in the Course of the Study. 
Table 3: Summary of the Occurrence of Different Types of Seizures Recorded for Patients for the 2 Month Interval Prior to the Study.

\begin{tabular}{lrrrr} 
Seizure Type & \multicolumn{2}{c}{$\begin{array}{c}\text { Number of Patients } \\
(\%)\end{array}$} & $\begin{array}{c}\text { Number of Seizures per Month } \\
\text { Range }\end{array}$ & Mean \pm S.D. \\
\hline CPS + SGS combined 114 & $(100 \%)$ & $1-4$ & $2.8 \pm 1.0$ \\
CPS & 111 & $(97 \%)$ & $0-4$ & $2.7 \pm 1.1$ \\
SGS & 31 & $(27 \%)$ & $0-4$ & $0.8 \pm 0.8$ \\
SPS & 32 & $(28 \%)$ & $0-100$ & $6.4 \pm 18.0$ \\
\hline
\end{tabular}

CPS - Complex Partial Seizures

SGS - Secondarily Generalized Seizures

SPS - Simple Partial Seizures

The effects of gabapentin treatment on the primary efficacy parameter, defined as the percentage of patients in whom a decrease of $50 \%$ or more in the combined frequency of CPS + SGS between baseline and endpoint is presented in Figure 2. Response to gabapentin treatment was observed in $71 \%$ of patients $(p=0.0001)$. During the treatment period, $46 \%$ of the patients were seizure-free. Similar results were observed when individual seizure types (CPS, SGS and SPS) were analyzed. A trend towards a higher response rate to gabapentin was observed in parameters with CPS, as compared to SGS and SPS (Figure 3 ). The response for individual seizure types ranged between $47 \%$ and $74 \%$, while the percentage of patients who were seizure-free at the end of the study varied between $31 \%$ and $55 \%$. The analysis of the response rate for individual seizure types reached a high statistical significance for CPS ( $p=$ $0.0001)$, but not for the SGS $(p=0.651)$ or SPS $(p=0.197)$ type.

In an attempt to better characterize the patient population in whom a decrease in seizure frequency of $50 \%$ or more was

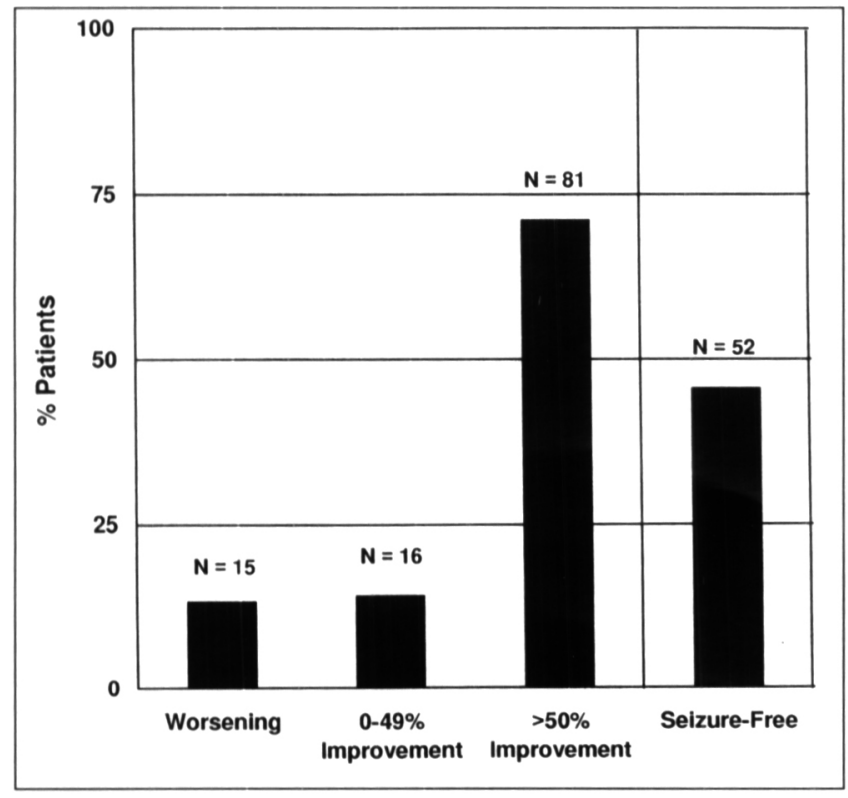

Figure 2: Changes in Combined Frequency of Complex Partial Seizures + Secondarily Generalized Seizures after 20 Weeks of Gabapentin (Neurontin (B) Therapy, Compared to Baseline, $N=114$. Response to treatment, a reduction of $50 \%$ or more in seizure frequency, was noted in $71 \%$ of patients $(p=0.0001)$.

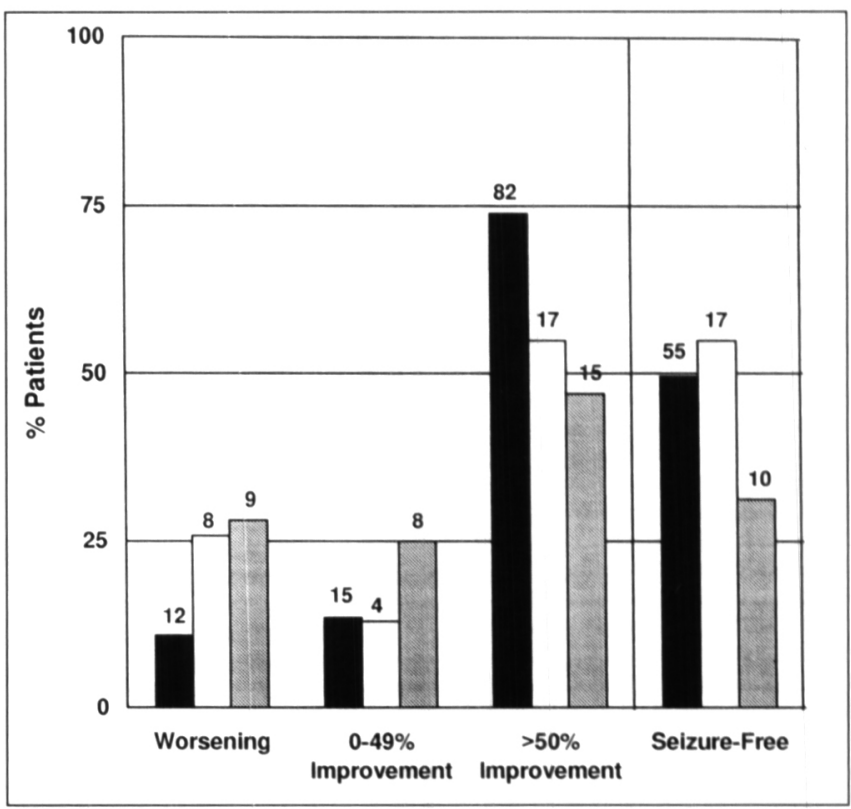

Figure 3: Changes in Frequency of Individual Seizure Types after 20 Weeks of Gabapentin (Neurontin $®)$ Therapy, Compared to Baseline. Complex Partial Seizures, $N=111^{*}(\square)$; Secondarily Generalized Seizures, $N=31^{*}(\square)$; Simple Partial Seizures, $N=32$ ( $\left.\square\right) ; p=$ 0.0001 for CPS.

*Data missing in 2 patients.

observed, data were stratified according to the patients' gender, duration of epilepsy, daily dose of gabapentin, or number of prior AEDs. None of these co-variables were correlated with the response to gabapentin (data not shown).

\section{Quality of Life Assessment}

Data from the QOLIE-10 evaluation were available at both baseline and after 20 weeks treatment in 103 to 109 patients depending on the question, except for Question 3 (impact of epilepsy or AEDs on driving), for which it was available in only 70 individuals. The average percent improvement observed in the individual questions ranged between 3 and $14 \%$, as shown in Figure 4. A highly statistically significant improvement $(\mathrm{p}=$ 0.0002) was observed for the "Composite QOL Score", which represented a combination of the scores from all 10 questions. Changes for individual questions reached statistical significance ( $\mathrm{p}<0.05$; paired t-test) for Questions 1 (energy level), 2 (feeling downhearted and blue), 5 (work limitations), 9 (fear of seizure), and 10 (overall quality of life). A positive trend was also observed in the mental effects of AEDs $(p=0.084)$.

\section{Maintenance Doses of Gabapentin}

The mean daily dose of gabapentin at the end of 20 weeks treatment was $1560 \pm 520 \mathrm{mg}$ (range $300-3200 \mathrm{mg}$ ). The maintenance dose was $1420 \pm 420 \mathrm{mg} /$ day in patients with a decrease of $50 \%$ or more in seizure frequency, and $1930 \pm 600 \mathrm{mg} /$ day in other patients, thus reflecting the individualized titration scheme which attempted to reach an effective and tolerable dose in each patient. The dose level did not show any significant relationship with age, sex or body weight. Dosage reduction from the maximum achieved dose occurred in 12 instances during the study. Except for one patient who became seizure-free with a dose of gabapentin of $800 \mathrm{mg} / \mathrm{day}$, all individuals with no seizures at the 


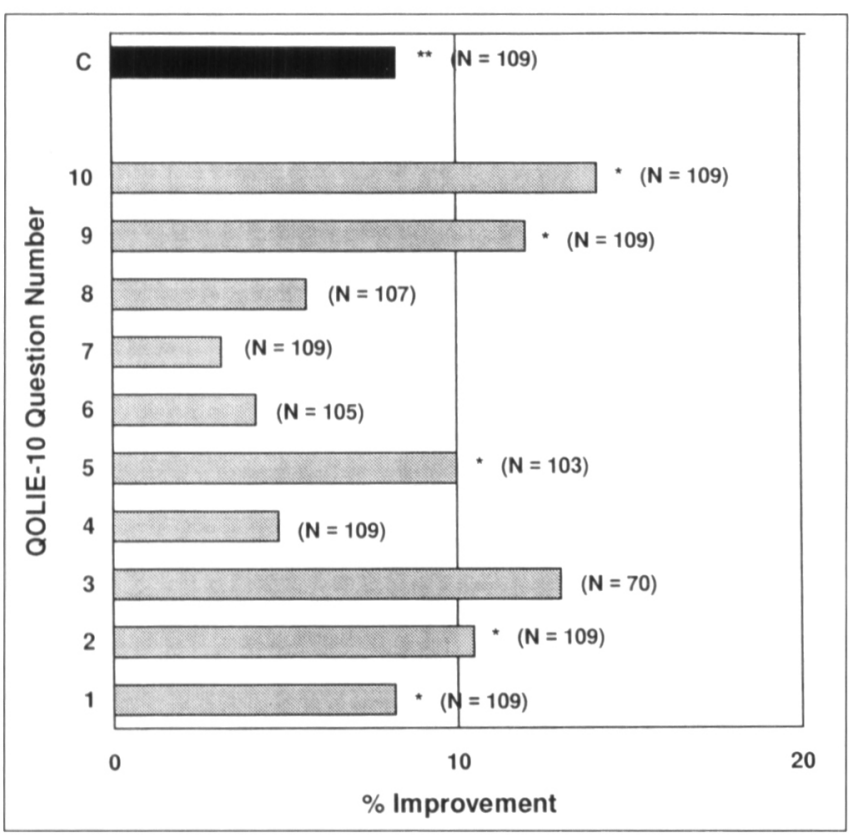

Figure 4: Improvement in Quality of Life Parameters for Study Patients $(N=114)$ after 20 weeks of Gabapentin (Neurontin $(B)$ Therapy Based on QOLIE-10 Evaluation. QOLIE-10 question numbers include: 1 = energy level: 2 = feeling downhearted \& blue; $3=$ effects of epilepsy or AED on driving; $4=$ memory difficulties; $5=$ work limitations; $6=$ social limitations; $7=$ physical effects of $A E D ; 8-; 9=$ fearful of seizures; $10=$ overall quality of life; ${ }^{*} p<0.05$. $C=$ composite score; ${ }^{* *} p=0.0002$.

endpoint evaluation were receiving a daily dose of $1200 \mathrm{mg}$ or more. Figure 5 presents further details on the distribution of the mean daily dose at the endpoint evaluation in the evaluable patient population $(\mathrm{N}=114)$.

\section{Safety Assessments}

Throughout the study 99 patients reported 251 adverse events. Each adverse event was judged by investigators with regard to its causal relationship to gabapentin. However, for those adverse events deemed probably or definitely related to gabapentin, no withdrawal/challenge tests were conducted. The most frequent drug-related adverse events ( $>5 \%$ frequency) included somnolence $(16 \%)$, dizziness $(9 \%)$, and asthenia $(6 \%)$. Table 4 outlines, for the intent-to-treat patient population $(\mathrm{N}=$ 136; data missing in 5 patients), the results of the overall assessment of patients' tolerability of gabapentin performed by the investigators at the end of the study. As shown, good to excellent tolerability was reported in more than three out of four (77\%) patients.

Table 4: Overall Assessment of Tolerability of Patients $(\mathrm{N}=141)^{*}$ to Gabapentin (Neurontin ${ }^{B}$ ) after 20 Weeks Treatment.

\begin{tabular}{|c|c|}
\hline Category & Number of Patients (\%) \\
\hline Excellent & $62(44 \%)$ \\
\hline Very good & $109(77 \%)$ \\
\hline Good & $21(15 \%)$ \\
\hline Fair & $6(4 \%)$ \\
\hline Poor & $21(15 \%)$ \\
\hline
\end{tabular}

*Data missing in 5 patients.

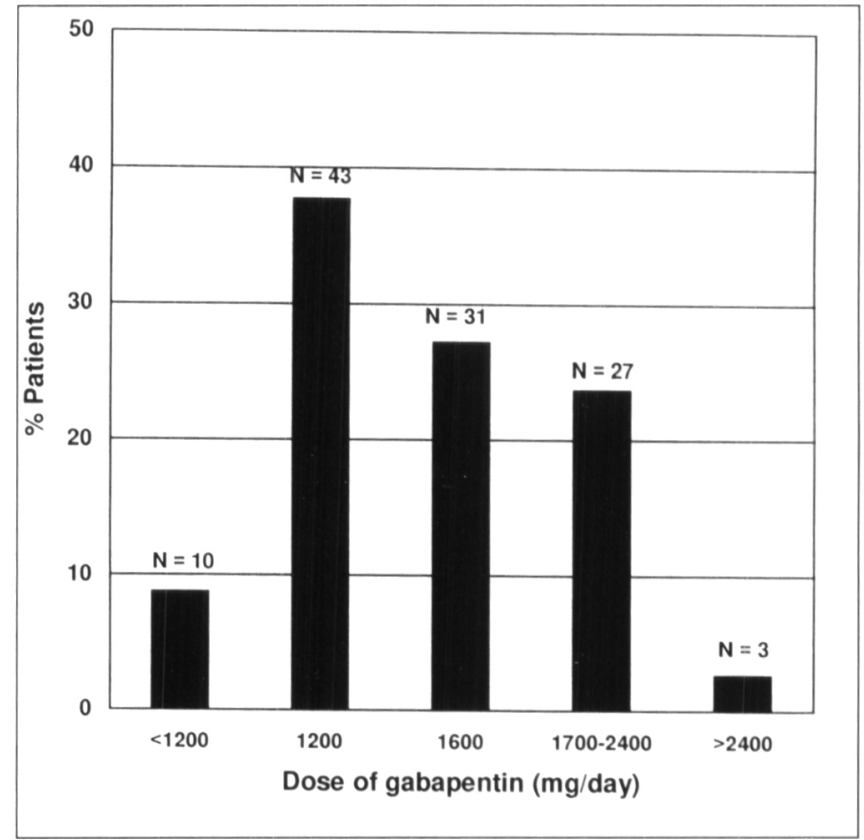

Figure 5: Range of Endpoint Gabapentin (Neurontin $\circledast$ ) Daily after 20 Weeks of Adjunctive Therapy, $N=114$. The mean dose of gabapentin was approximately $1600 \mathrm{mg} /$ day.

\section{Neurological Examination}

Nystagmus was observed in 9 patients at the baseline visit and in 7 at the endpoint evaluation, including 3 patients in whom it was present at both time points. No other remarkable findings were observed in the neurological examination.

\section{Clinical Laboratory Evaluations}

Only one clinically significant abnormality, namely, the presence of leucocytes in urine, was observed in one patient at the end of the study.

\section{Discussion}

Standard AEDs are used as first treatment for patients newly diagnosed with epilepsy despite concerns that exist with regard to their potential toxicity, their possible interactions with other drugs, and the need for regular blood level monitoring. Although the drugs recently introduced for the treatment of epileptic seizures are generally regarded as potentially presenting advantages over the standard AEDs, ${ }^{1} 11$ these are typically approved as adjunctive management of epilepsy for patients not satisfactorily controlled with conventional therapy.

Gabapentin possesses a pharmacokinetic profile with many of the features of the ideal AED. This drug is not metabolized before excretion, is not protein-bound, does not induce hepatic enzymes, and, in contrast to other established or new AEDs, does not interact with common AEDs such as phenytoin, carbamazepine, valproate, or phenobarbital. In line with these expectations, gabapentin has been evaluated in over 3,000 patients since the initiation of its clinical development ${ }^{13,4,7,8}$ and has been confirmed to be well tolerated.

Most safety and efficacy studies reported to date on gabapentin were conducted in severe cases of epilepsy. Despite the fact that these patients represented a difficult-to-treat population, these 
studies demonstrated the efficacy and high safety profile of gabapentin as adjunct therapy for partial seizures and secondarily generalized seizures. ${ }^{3.7,8}$ Importantly, serious reactions have been extremely rare, in contrast to other newly introduced AEDs, such as lamotrigine, with which severe cases of rash may be observed, or vigabatrin, for which instances of depression and psychiatric symptoms have been reported. ${ }^{2,3}$

The present study was aimed at collecting information in conditions as close as possible to the regular environment under which the individual patients' seizures were being treated and managed at their respective clinics. The major objective was to assess the potential of gabapentin as adjunct to conventional AEDs in patients less severely affected with epilepsy than in the typical refractory case studies. However, the patient population that was enrolled turned out to be more severely affected than initially expected. Patients had been suffering from epilepsy for an average of 20 years, and more than $75 \%$ had previously failed on AEDs other than CBZ or PHT. Notwithstanding the fact that this trial was conducted under an open-label design, the present results strongly indicate the therapeutic benefit of gabapentin as first add-on to CBZ and/or PHT. In view of the fact that $46 \%$ of patients were seizure-free after gabapentin treatment, this is unlikely to be explained by a regression towards the mean or to non-drug effects. A high response rate was reported for all seizure types, and CPS in particular. Frequency of CPS + SGS decreased by $50 \%$ or more in over $70 \%$ of the patients. Even taking into account the possibility of a nonpharmacological study effect which could not be assessed in the absence of a control group, the fact that half of the patients reported to be seizure-free during the last 8 weeks of the treatment period is indicative of a high efficacy of gabapentin in the patient population studied. The response rate for SGS and SPS when analyzed individually did not reach statistical significance due to substantially fewer patients with these seizures, even though reductions in the number of each seizure type were noted. In addition, there were a number of patients (8-12) in individual seizure categories who did not improve but worsened during the course of the study. The reason for the increase in seizure frequency is not known. However, the pharmacokinetic profile of gabapentin precludes drug interactions with $\mathrm{CBZ}$ or PHT. $1.3,4.7 .8$

The assessment of quality of life also showed positive outcomes during the treatment with gabapentin. This positive effect was observed in all scores generated by the individual questions of the QOLIE-10 questionnaire. A statistically significant improvement was reached for questions related to energy level, feeling downhearted and blue, work limitations, fear of seizure occurrence and overall quality of life. In addition, the "Composite QOL Score" combining all 10 questions of the QOLIE-10 showed a statistically significant improvement. These findings are in further support of the beneficial effects of gabapentin in terms of both seizure control and overall tolerability.

Premature discontinuation related to adverse events was in the same range as reported in previous controlled trials, ${ }^{12}$ and the events that led to discontinuation were all isolated in nature. Similarly, the incidence of the adverse events most frequently observed was in the same range or lower than in previous controlled trials with gabapentin. ${ }^{2}, 12$ No significant, drug-related adverse events were reported. The high tolerability profile of gabapentin is further substantiated by the fact that a good to excellent tolerability was reported for more than $75 \%$ of the patients in the overall assessment conducted by the investigators.

Management of epilepsy in elderly patients is an increasing clinical concern, and requires understanding of their unique biochemical and pharmacological characteristics including greater susceptibility to adverse side effects, concomitant illnesses that require multiple medications, decreased gastrointestinal function, decreased body water, decreased plasma concentration, renal and hepatic function. ${ }^{13}$ The present study included 11 patients over 65 years of age. These patients benefited from gabapentin add-on therapy, with good tolerability and without adverse effects. These observations support recent clinical and pharmacokinetic studies that demonstrate benefits of gabapentin in the elderly because it is rapidly absorbed, is water soluble, is not metabolized by the liver, shows no drug interactions with other AEDs and the dosage may be adjusted readily based on renal function. ${ }^{14,15}$

Comparison of the present findings with other new AEDs is limited currently due to the lack of direct comparative data in a similar patient population and with a similar experimental design. However, as suggested in a recent European pharmacoeconomic study, ${ }^{16}$ the overall profile of gabapentin in terms of efficacy, safety and ease of use from a pharmacokinetic standpoint makes it an ideal choice as adjunctive therapy to conventional AEDs in adult epileptic patients with partial seizures.

The long term efficacy and safety of gabapentin also has been demonstrated in clinical trials and in clinical practice. The present study followed a period of 20 weeks treatment. However, in a follow-up to the present study, ${ }^{17.18}$ at 12 months or more of adjunctive gabapentin treatment conducted in 49 patients, $69 \%$ of patients experienced $\geq 50 \%$ reduction in the frequency of complex partial seizures with or without secondary generalization, a responder rate comparable to that presented here. Additionally, $51 \%$ of patients were seizure-free at followup evaluation. The improvement in the quality of life observed at 12 months or more of treatment was comparable to that observed at 20 weeks. The good safety profile of gabapentin adjusted by the low incidence of adverse events, the good tolerability of adjunctive gabapentin therapy and the neurological examination at 5 months was sustained, with no evidence of tolerance developing to gabapentin.

As this trial was conducted under normal conditions of clinical practice with minimal interference in the regular management of epileptic patients, these data provide a valuable guide to the use of gabapentin in neurological practice for patients on conventional therapy that are judged in need of the addition of a new AED for the management of their epileptic seizures.

\section{APPENDIX}

NEON (Neurontin Evaluation of Outcomes in Neurological Practice) Study Investigators Group: Contributors

$\dagger M$. Aubé, Montreal Neurological Institute, Montreal, Quebec; S. Bedwell, Halifax, Nova Scotia; D. Bergeron, Rimouski, Quebec; S.C.K. Cheung, Sudbury, Ontario; J. Chu, Etobicoke, Ontario; J.-M. Côté, Hôtel-Dieu du Sacré-Coeur de Jésus, Quebec City, Quebec; R. Delisle, Trois-Rivières, Quebec; H.B. Desai, Windsor Health Unit, Windsor, Ontario; E. Deutsch-Andermann, Montreal Neurological Institute, Montreal, Quebec; F. Dominique, Winnipeg, Manitoba; S. Fawcett, Hamilton, Ontario; D. Hallé, Hull, Quebec; K.J. Ho, Kitchener, Ontario; B.H. 
Jones, Kelowna, British Columbia; D.B. King, Halifax, Nova Scotia; J. Lachapelle, Hôpital Maisonneuve-Rosemont, Montreal, Quebec; M.-A. Lee, Foothills Hospital, Calgary, Alberta; R. Lo, Hamilton, Ontario; V. Makin, North Vancouver, British Columbia; S. McKenzie, Mississauga, Ontario; R.S. McLachlan, University Hospital, London, Ontario; G. Moddel, Scarborough, Ontario; C. Nair, Regina, Saskatchewan; D. Novak, Penticton, British Columbia; G. Patry, Hôtel-Dieu du SacréCoeur de Jésus, Quebec City, Quebec; N. Pillay, Health Sciences Centre, Winnipeg, Manitoba; P. Ranalli, Downsview, Ontario; D. Silverberg, Moncton, New Brunswick; L.D. Sitwell, Ottawa, Ontario; E. Starreveld, Hys Medical Center, Edmonton, Alberta; M. Thibault, Hôtel-Dieu du Sacré-Coeur de Jésus, Quebec City, Quebec; P. Tovich, Peterborough, Ontario; F. Veloso, Pasqua Hospital - The Medical Centre, Regina, Saskatchewan; D.F. Waller, Oshawa, Ontario; S. Wiebe, University Hospital, London, Ontario; M. Winger, Windsor, Ontario.

\section{ACKNOWLEDGEMENTS}

The contributions of Nina Maron, Ph.D. and Renata Szczepanska, M.Sc. are gratefully acknowledged. This study was supported by a grant from Parke-Davis, Canada.

\section{REFERENCES}

1. Mattson RH. Efficacy and adverse effects of established and new antiepileptic drugs. Epilepsia 1995; 36 (Suppl. 2): S13-S26.

2. á Rogvi-Hansen B, Gram L. Adverse effects of established and new antiepileptic drugs: an attempted comparison. Pharmacol Ther 1995; 68(3): 425-434.

3. Dichter MA, Brodie MJ. New antiepileptic drugs. N Engl J Med 1996; 334(24): 1583-1590.

4. Leach JP, Brodie MJ. New antiepileptic drugs - an explosion of activity. Seizure 1995; 4: 5-17.
5. Andrews CO, Fisher JH. Gabapentin: a new agent for the management of epilepsy. Ann Pharmacother 1995; 28: 1188-1196.

6. Taylor CP. Emerging perspectives on the mechanism of action of gabapentin. Neurology 1994; 44 (Suppl. 5): S10-S16.

7. Bruni J. Titration of gabapentin dose for optimal control of epileptic seizures. Adv Therapy 1996; 13(6): 324-334.

8. Bruni J. Gabapentin. Can J Neurol Sci 1996; 23 (Suppl. 2): S10S12.

9. Proposal for revised Classification of epilepsies and epileptic syndromes. Commission on Classification and Terminology of the International League against Epilepsy. Epilepsia 1989; 30(4): 389-399.

10. Devinsky O, Cramer JA and the QOLIE Development Group. Professional Postgraduate Services, a division of Physicians World Communications Group, Secaucus, NJ, USA.

11. Wilder BJ. The treatment of epilepsy: an overview of clinical practices. Neurology 1995; 45 (Suppl. 2): S7-S11.

12. Ramsay RE. Gabapentin: Toxicity. In: Levy RH, Mattson RH, Meldrum BS, eds. Antiepileptic Drugs, 4th Ed. New York: Raven Press, 1995; 857-860.

13. Willmore LJ. The effect of age on pharmacokinetics of AED's. Epilepsia 1995; 36 (Suppl. 5): S14-S21.

14. McLean MJ. Clinical pharmacokinetics of gabapentin. Neurology 1994; 44 (Suppl. 5): S17-S22.

15. Perucca E. Pharmacokinetic profile of topiramate in comparison with other new AED's. Epilepsia 1996; 37 (Suppl. 2): S8-S13.

16. Hughes D, Cockerell OC. A cost minimization study comparing vigabatrin, lamotrigine and gabapentin for the treatment of intractable partial epilepsy. Seizure 1996; 5: 89-95.

17. Bruni J. Neurontin $®$ as add-on therapy in patients with partial seizures - long term study Proc. World Congress of Neurology [abstract] (in press).

18. Bruni J. Efficacy of gabapentin (Neurontin®) as first add-on therapy in the treatment of partial seizures - long term follow-up [abstract] Epilepsia 1997; 38 (Suppl. 8): S94. 